\title{
Numerical and Analytical Modeling of Aligned Short Fiber Composites including Imperfect Interfaces
}

\author{
J.A. Nairn and M. Shir Mohammadi* \\ Wood Science and Engineering, Oregon State University, Corvallis, OR 97330, USA
}

\begin{abstract}
Finite element calculations were used to bound the modulus of aligned, short-fiber composites with randomly arranged fibers, including high fiber to matrix modulus ratios and high fiber aspect ratios. The bounds were narrow for low modulus ratio, but far apart for high ratio. These numerical experiments were used to evaluate prior numerical and analytical methods for modeling short-fiber composites. Prior numerical methods based on periodic boundary conditions were revealed as acceptable for low modulus ratio, but degenerate to lower bound modulus at high ratio. Numerical experiments were also compared to an Eshelby analysis and to an new, enhanced shear lag model. Both models could predict modulus for low modulus ratio, but also degenerated to lower bound modulus at high ratio. The new shear lag model accounts for stress transfer on fiber ends and includes imperfect interface effects; it was confirmed as accurate by comparison to finite element calculations.
\end{abstract}

Keywords: A. Discontinuous reinforcement, B. Interface/interphase, B.Elasticity, C. Finite element analysis (FEA)

\section{Introduction}

In mean-field modeling of short-fiber composite materials, a composite unit cell is subjected to mean stress or strain and the effective stiffness or compliance tensors are found by averaging strains and stresses throughout the composite [1]. This averaging is done over all unit cell orientations using a fiber orientation distribution function. The unit cell for this analysis is a short fiber composite with all fibers aligned in the same direction. Thus, the fundamental problem for analysis of short-fiber composites is to determine mechanical properties of an aligned, short-fiber composite.

\footnotetext{
*Corresponding author, john.nairn@oregonstate.edu
} 
One might think this problem is solved by methods such as Eshelby [2], Mori-Tanaka [3], modern shear-lag models [4-7], or numerical methods [8-13], but some gaps appear. First, most prior numerical studies have been limited to modest fiber/matrix modulus ratios of $R=E_{f} / E_{m}<30$ and relatively short fiber aspect ratios, $\rho=l_{f} / d_{f}<30[8,9,11,13]$. Gusev and Lusti $[10,12]$ looked at higher aspect ratios, but only for a narrow selection of $R$ and fiber volume fraction, $V_{f}$. As a consequence, the validation of analytical models by these numerical studies [9] only validates them for the corresponding small range of properties.

A recent trend in composites research, especially in nanocomposites, is to reinforce soft polymers (e.g., elastomers with $R>10^{4}$ ) and isolate nano-fibers with aspect ratios higher then 30 [14-17]; the results of such work has been a challenge to model. Figure 1 show some experimental results for reinforcement of an elastomer with nano-cellulose fibers [14] and compares them to an existing analytical model (labeled "Mori-Tanaka" [3]) and an existing numerical method based on large periodic representative volume elements (RVEs) with randomly placed fibers (labeled "Periodic RVE (FEA)" using approach of Gusev [8]). These experimental results are two to three orders of magnitude higher then existing models. The question arises - are these high reinforcements the discovery of a new nanophenomenon that cannot be modeled with continuum mechanics or do continuum methods just need to be revised for high $R$ ? To explore this question, we developed a new numerical method to derive upper and lower bounds to the modulus. The sample calculation of bounds in Fig. 1 (see dashed lines) shows that experimental results fall within continuum mechanics bounds and that prior modeling methods all degenerate to lower bound results. In other words, the methods described here have new potential to guide expectations of properties for composites with high $R$.

To study composite modeling methods at high $R$ and aspect ratio as well has how they relate to conventional methods at low $R$ and aspect ratio, we ran numerical calculations for a very wide range of $R$ (from 10 to $10^{5}$ ) and aspect ratios (from 5 to 100). The calculations in this part of the study were based on novel methods that allowed us to numerically determine upper and lower bounds to the fiber-direction modulus. The shear number of calculations along with the size of mesh (particularly at high aspect ratio) precluded mesh refinement of numerical results. A powerful feature of the bounding method, however, is that it allows one to get definitive bounds even without mesh convergence. These numerical results provided input for considering four questions:

What is correct modulus? One of the best ways to judge the accuracy of modeling methods is to compare them to numerical results [9], but what numerical method gives the correct modulus? Here we derived 


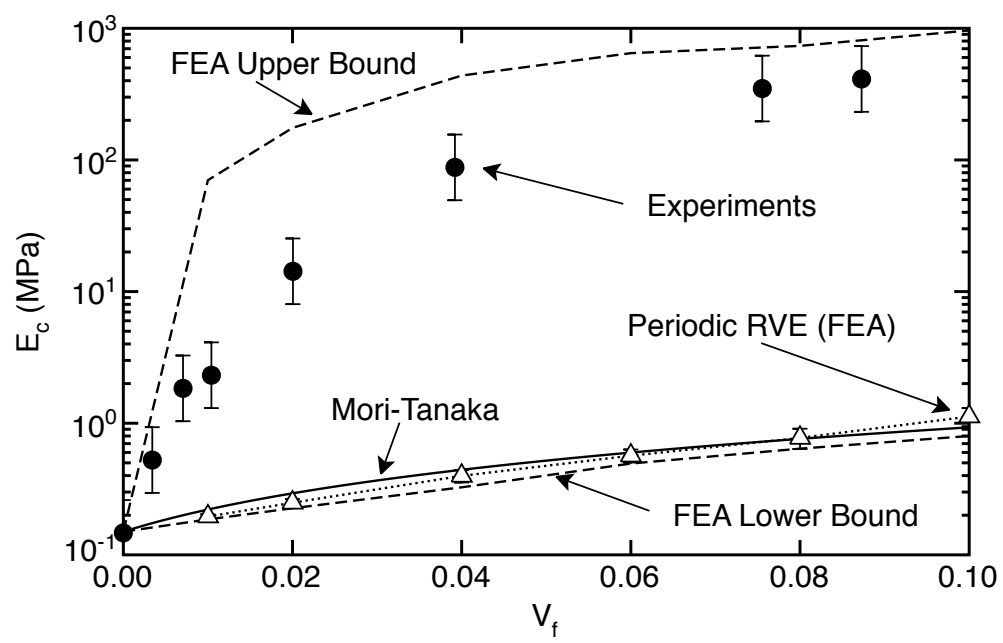

Figure 1: The symbols are experimental results from Ref. [14] with $R=9.5 \times 10^{5}$, which are compared to existing modeling methods (Mori-Tanaka and Periodic RVE (FEA)) and to upper and lower bounds described in this paper (dashed lines). The experiments are quasi-2D with fibers claimed to be randomly aligned in the plane of a film. The models are 2D calculations for aligned fibers. The comparison with experiments is only qualitative, but if experiments had aligned fibers, they would move toward the upper bound and still demonstrate that prior models are near the lower bound and far below experiments.

numerical bounds using Monte Carlo methods with randomly placed and well-dispersed, aligned fibers. We used bounding methods to define limits on the modulus for $R$ up to $10^{5}$ and $\rho$ up to 100 . The separation of the bounds shows that the calculated modulus depends on boundary conditions, especially for large $R$.

How do periodic RVE calculations compare to numerical bounds? Most numerical models use periodic RVEs and assume analysis results with periodic boundary conditions are equivalent to bulk composite properties. To test this hypothesis, we compared the new numerical bounds to both small periodic RVEs (using either cylindrical (rectangles in 2D) or elliptical fibers) and large RVEs with random fibers. All periodic RVE methods work well for low $R$, but degenerate to lower bound results at high $R$.

Can an analytical model sufficiently capture the results of periodic RVE composites? Given the capabilities (and limitations) of periodic RVE analysis, an analytical model that agrees with those numerical results would have those same capabilities (and limitations). We developed an improved shear-lag model for short composite fibers that explicitly includes stress transfer on the fiber ends and imperfect interfaces. The new model, along with an Eshelby [2] analysis, were compared to numerical results on the same geometries. These analytical methods can reproduce numerical methods based on periodic conditions, which means they give good prediction for low $R$, but degenerate to lower bound results for high $R$. 
Can an analytical model account for 3D fibers and for imperfect interfaces? The first three questions used 2D calculations and assumed perfect fiber/matrix interfaces. Real composites are 3D and may have imperfect interfaces. We lastly considered 3D single fiber RVE results by comparing axisymmetric numerical calculations with imperfect interfaces to the new shear lag analysis with concentric cylinders that also includes imperfect interface effects. The new model accurately reproduces all numerical results including the role of imperfect interfaces.

\section{Methods}

All finite element calculations (FEA) were linear elastic, static, and two dimensional. Most simulations were plain strain analyses although some 3D results were generated using axisymmetric simulations. All calculations were done using the open source code NairnFEA [18] with 8-node quadrilateral elements. Issues involving convergence are discussed in section 3. By using script control, we automated the thousands of FEA calculations needed to get sufficient results for answering the posed questions. The FEA calculations were run on either desktop computers or Linux nodes in a cluster. The main requirement for the largest calculations was to have sufficient memory (more than $5 \mathrm{~GB}$ ).

\section{Results and Discussion}

\subsection{What is the Correct Modulus?}

To run numerical experiments for the "correct" modulus of aligned short fiber composites, we ran FEA calculations on representative composites with randomly placed fibers. The fibers were all aligned in one direction, placed using a random sequential adsorption (RSA) method [11], and well dispersed (separated by at least one element in the mesh). The numerical experiments were done for fiber to matrix modulus ratios of $R=10,100,1000,10^{4}$, and $10^{5}$, for fiber aspect ratios of $\rho=5,10,20,40$, 70 , and 100, and for fiber volume fractions of $V_{f}=0.01,0.02,0.05,0.1,0.15,0.2$, and 0.25 . Monte Carlo methods were used to account for the random structures. For each combination of $R, \rho$, and $V_{f}$, we ran FEA calculations for 20 random structures and averaged the results for mean and standard deviation of the modulus. For most property settings, the 20 replicates gave sufficiently narrow errors bars on the results. The total number of FEA calculations required to map the parameter space exceeded 15,000 .

The first issue was the mesh. To deal with randomly placed fibers with randomly situated stress concentrations, the modeling used a regular mesh. A quick calculation showed that a 3D mesh for 
the largest aspect ratio would have over a billion degrees of freedom, which is infeasible for the 15,000 calculations we needed to run. 3D calculations by Gusev [8] required 30 processor-hours per calculation and that was for spherical inclusions ( $\rho=1$ ) which can use much smaller RVEs then needed here. We therefore switched to 2D, plain-strain FEA (which still can be used to evaluate other methods provided comparisons are made to 2D versions of those methods). Even in 2D, the mesh could not be highly refined. We used the crudest mesh possible where the element size was equal to the fiber diameter. Thus each fiber had one element across its width and the well-dispersed fibers were separated by at least one fiber diameter (i.e., one mesh element). With this mesh, the largest calculation had about 200,000 degrees of freedom and could be completed in a 5-30 minutes (depending on computer speed).

Because we were limited to a crude mesh, we could not refine the mesh for convergence. To allow definitive results with such a mesh, we adopted a bounding method. In composite variational mechanics, upper and lower bound results are found by the solving the two problems in Fig. 2 [1, 1921]. First, the composite is subjected to constant tractions, $\boldsymbol{T}$, over the entire surface of

$$
T=\sigma_{0} \cdot \hat{n}
$$

where $\boldsymbol{\sigma}$ is the uniform applied stress and $\hat{n}$ is surface normal. For stress corresponding to axial loading in the fiber direction (see Fig. 2A), the complementary energy, as approximated by FEA strain energy $\left(\Gamma_{F E A}\right)$, must be greater than or equal to the exact complementary energy, $\Gamma$, leading to

$$
\Gamma_{F E A} \geq \Gamma=\frac{\sigma_{0}^{2} V}{2 E_{y y}^{(r)}} \quad \text { or } \quad E_{y y}^{(r)} \geq \frac{\sigma_{0}^{2} V}{2 \Gamma_{F E A}}=E_{L B}
$$

where $V$ is specimen volume and $E_{y y}^{(r)}$ is the effective, plain strain modulus in the $y$ direction. In other words, an FEA calculation of $\Gamma_{F E A}$ provides a lower bound to the axial modulus $\left(E_{L B}\right)$.

Second, the composite is subjected to a uniform strain field, which corresponds to fixed displacement (in both directions), $\boldsymbol{u}$, over the entire surface of

$$
u=\varepsilon_{0} \cdot x
$$

where $\boldsymbol{\varepsilon}_{0}$ is the applied strain and $\boldsymbol{x}$ is position on the surface. For such fixed-strain conditions (see Fig. 2B), the FEA strain energy $\left(U_{F E A}\right)$ is a rigorous upper bound to the exact strain energy, $U$, leading to

$$
U_{F E A} \geq U=\frac{1}{2} \varepsilon_{0} \cdot C \varepsilon_{0} V
$$

where $C$ is the effective stiffness tensor. Numerical calculation of axial modulus when using fixed-strain boundary conditions requires three separate FEA calculations: 


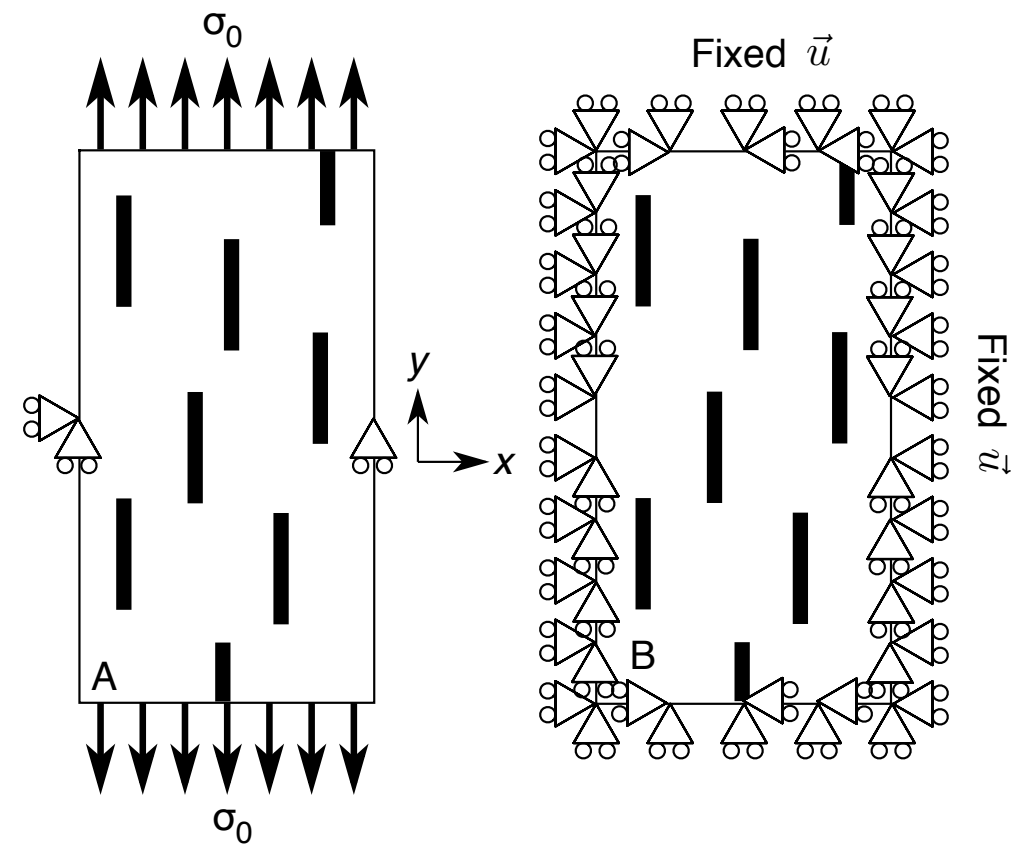

Figure 2: Schematic view of finite element model used for stress and fixed-strain boundary conditions. A. Stress boundary conditions apply uniform stress in the y direction on the top and bottom edges and no stress on the side edge. A minimal number of displacement conditions are used to prevent rigid body rotation. B. Fixed-strain boundary conditions apply fixed displacements in both the $x$ and $y$ directions on all edges to match the imposed strains. 
1. Use $\varepsilon_{x}=\varepsilon_{0}, \varepsilon_{y}=0$, and $\gamma_{x y}=0$. An FEA calculation gives $C_{11} \leq 2 U_{11} /\left(\varepsilon_{0}^{2} V\right)$ (where $U_{11}$ is the strain energy from that FEA analysis).

2. Use $\varepsilon_{x}=0, \varepsilon_{y}=\varepsilon_{0}$, and $\gamma_{x y}=0$. An FEA calculation gives $C_{22} \leq 2 U_{22} /\left(\varepsilon_{0}^{2} V\right)$.

3. Use $\varepsilon_{x}=\varepsilon_{0}, \varepsilon_{y}=\varepsilon_{0}$, and $\gamma_{x y}=0$. An FEA calculation gives $C_{11}+C_{22}+2 C_{12} \leq 2 U_{12} /\left(\varepsilon_{0}^{2} V\right)$.

A reduced plain-strain axial modulus can be found from these three results using

$$
E_{y y}^{(r)} \leq \frac{2}{\varepsilon_{0}^{2} V}\left[U_{22}-\frac{\left(U_{12}-U_{11}-U_{22}\right)^{2}}{4 U_{11}}\right]=E_{U B}
$$

The plain strain properties are defined from the reduced compliance tensor, $\mathrm{S}^{(r)}$, which is the inverse of the stiffness tensor found by 2D FEA:

$$
\left[\begin{array}{ccc}
C_{11} & C_{12} & 0 \\
C_{12} & C_{22} & 0 \\
0 & 0 & C_{66}
\end{array}\right]^{-1}=\mathrm{S}^{(r)}=\left[\begin{array}{ccc}
1 / E_{x x}^{(r)} & -v_{x y}^{(r)} / E_{x x}^{(r)} & 0 \\
-v_{x y} / E_{x x}^{(r)} & 1 / E_{y y}^{(r)} & 0 \\
0 & 0 & 1 / G_{x y}^{(r)}
\end{array}\right]
$$

A side benefit of the three upper bound calculations is that they can also determine $E_{x x}^{(r)}$ and $v_{x y}^{(r)}$. One more calculation with $\varepsilon_{x}=\varepsilon_{y}=0$, and $\gamma_{x y}=\gamma_{0}$ can add the fourth (and final) in-plane property, $G_{x y}^{(r)}$. The results here focus on $E_{y y}^{(r)}$ although some comments on other properties are at the end.

Formally, neither the lower bound in Eq. (2) nor the upper bound in Eq. (5) are rigorous bounds. A rigorous lower bound requires complementary energy. Here we assumed strain energy found under stress boundary conditions is a good approximation to complementary energy. Although fixed-strain conditions give rigorous upper bounds to the elements of C, Eq. (5) combines those bounds to find a modulus that may not be a rigorous upper bound to axial modulus. Because the second term in Eq. (5) is generally small ( $<2 \%$ of $U_{22}$, especially at higher $R$ and $V_{f}$ ), $E_{y y}^{(r)}$ is effectively a rigorous upper bound property. We therefore took these two results as defining bounds for the composite modulus.

The next issue was the size required for the BVE. It should be large enough that results are unaffected by its size, but small enough to keep calculation times reasonable. Figure $3 A$ plots $E_{L B}$ for fixed traction (solid curves) and upper bound $C_{11}$ for fixed strain (dashed curves) as a function of the length of the BVE relative to the fiber length $\left(L / L_{f}\right)$ for fixed width of 20 times the fiber diameter $\left(d_{f}\right)$. These results are for fiber volume fraction $V_{f}=10 \%$, modulus ratio $R=10^{5}$ (more details on fiber properties are given below), and two fiber aspect ratios ( $\rho=10$ and 100); they include the extreme cases with the largest value of $R=10^{5}$ and largest $\rho=100$. Figure 3B shows analogous results as function of width of the BVE relative to fiber diameter $\left(W / d_{f}\right)$ for fixed length of $10 L_{f}$ and a different $R=10^{2}$. For RVE 

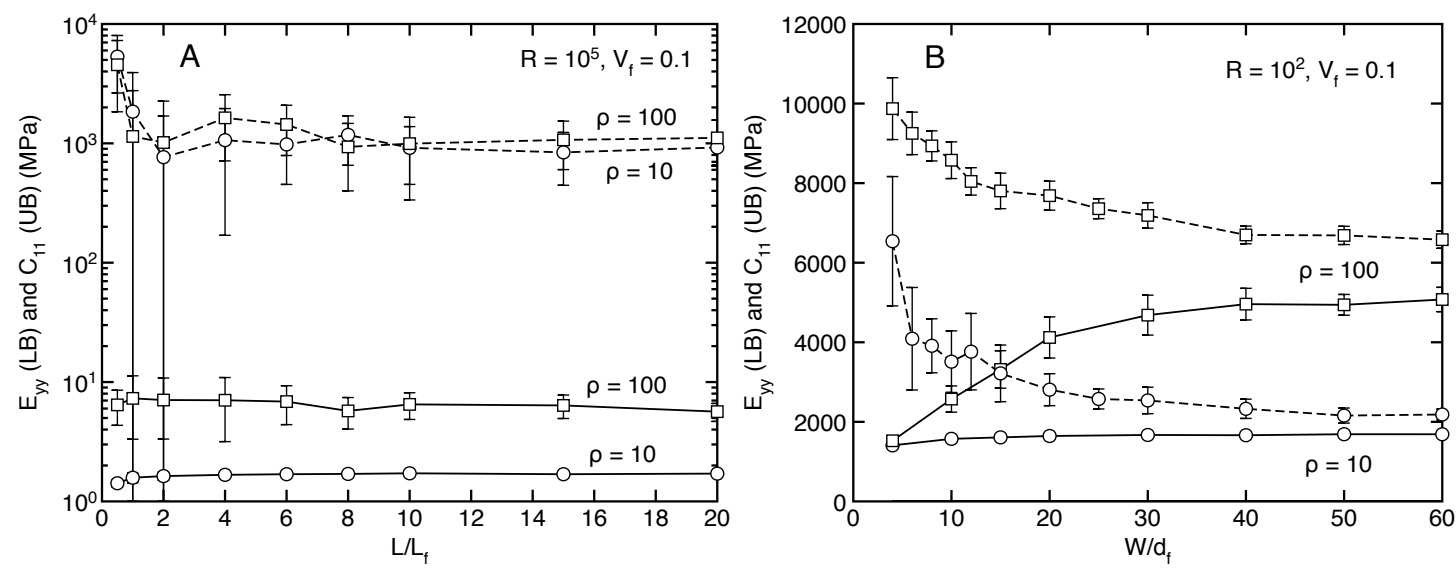

Figure 3: A. The lower bound axial composite modulus $E_{y y}(L B)$ and upper bound $C_{11}$ as a function of RVE length $\left(L / L_{f}\right)$ for fixed width of $20 d_{f}$ and for two fiber aspect ratios. B. The lower bound axial composite modulus $E_{y y}(L B)$ and upper bound $C_{11}$ as a function of RVE width $\left(W / d_{f}\right)$ for fixed length of $10 L_{f}$ and for two fiber aspect ratios. Both plots used fiber volume fraction $V_{f}=0.1$.

with short $L$, it is likely for fibers to span the entire length of the RVE resulting in an artificially high modulus that approaches the continuous fiber composite. As $L$ increased, the modulus decreased and plateaued above $L / L_{f}$ in the range of 5 to 10. Similarly, the magnitude of the error bars decreased up to about $L / L_{f}=10$. All subsequent simulations used $L / L_{f}=10$. For short width BVE, the modulus bounds were widely separated, but moved closer as $W$ increased. They plateaued at about $W / d_{f}=40$. Width effects at higher $R=10^{5}$ showed a small amount of continued decrease in the upper bound result for $W / d_{f}>40$. The decrease, however, was relatively small. Because using larger $W$ made simulations impractical, all subsequent simulations used $W / d_{f}=40$. Note that this BVE size is larger than some published RVE results. For example, Gusev and Lusti $[10,12]$ used $L / L_{f} \sim 3$ and $W / d_{f} \sim 12$. We expect that the nature of fixed strain boundary conditions, as opposed to periodic boundary conditions used in other FEA methods, is the cause of the need for BVEs to be larger than other RVEs.

Remark 1: Traditional RVE methods (e.g., [10-13]) use periodic boundary conditions where displacements normal to an edge are constant (such that plane sections remain plane) while tangential displacements are a degree of freedom [9]. These prior boundary conditions are neither fixed strain nor fixed traction. While they may converge to a result, they provide no information on where they fall relative to upper and lower bounds (i.e., how those boundary conditions affect the converged result). The boundary conditions used here to get bounds are different. The sample calculations in Fig. 1 suggest that traditional methods degenerate to lower bound results, while the bounding method has the poten- 
tial to bracket experimental results. To distinguish the fixed strain or traction boundary conditions used here from traditional RVE methods, the new method will be called a BVE method for bounded volume element.

Remark 2: Although a BVE model can formally describe a periodic structure, it seems unrealistic to assume fixed strain on the edge of a full-scale composite would translate to fixed strains on representative subelements of the composite. Instead, the BVE method is best imagined as external boundary conditions on a full-scale composite (albeit, a small one). When imagining a full-scale composite, it could be inconsistent to require the BVE to be geometrically periodic (as commonly done for RVEs of traditional methods [10]). Nevertheless, we compared geometrically non-periodic BVE calculations to some periodic ones and the differences were negligible for the size BVEs used.

Using the above method and BVE size, we ran all combinations of $R, \rho$, and $V_{f}$; some selected results are discussed here (other results are in subsequent sections). All simulations used an isotropic, elastic, high-modulus fiber with reduced plain strain modulus $E_{f}^{(r)}=100,000 \mathrm{MPa}$ and Poisson's ratio $v_{f}^{(r)}=0.33$ (the unreduced properties were $E_{f}=93,843 \mathrm{MPa}$ and $v_{f}=0.2481$ ). The plane strain matrix modulus varied as $E_{m}=E_{f} / R$ and its Poisson's ratio was the same as the fiber's. Figure 4A shows axial modulus for $R=100$ for three selected aspect ratios. The dashed curves are the lower bound and the solid curves are the upper bound. As expected, the modulus increased as volume fraction increased, but the increase is non-linear. The initial slope is low indicating low volume fraction does a relatively poor job of providing modulus. The slope gradually increased indicating increased fiber effectiveness at higher volume fraction. The error bars are rather small - in most cases smaller than the size of symbols used in the plots. Figure $4 \mathrm{~B}$ shows the same results for $R=10^{5}$ (on a log scale). Unlike the $R=100$ results, these results have lower bound modulus that is three orders of magnitude below the upper bound. Furthermore, the upper bound results have higher error bars; the coefficient of variation was over $100 \%$ at low $V_{f}$, but decreased to a range of $40 \%$ to $20 \%$ for $V_{f}$ from 0.15 to 0.25 .

Differences between upper and lower bounds in analytical modeling are well known to increase as $R$ gets larger [1]. But those differences are attributed to approximations in the modeling while numerical results are commonly assumed to hone in on the correct answer and therefore upper and lower bounds should be the same. The question remains - are the differences between upper and lower bounds because the FEA is not converged or something else? To answer this question, we refined the mesh. We could most refine the mesh for the shortest fiber with $\rho=5$. Figure 5 gives modulus as a function of mesh element size for $R=100$ and for $R=10^{5}$ when $\rho=5$ and $V_{f}=0.25$. As the mesh was refined, the 

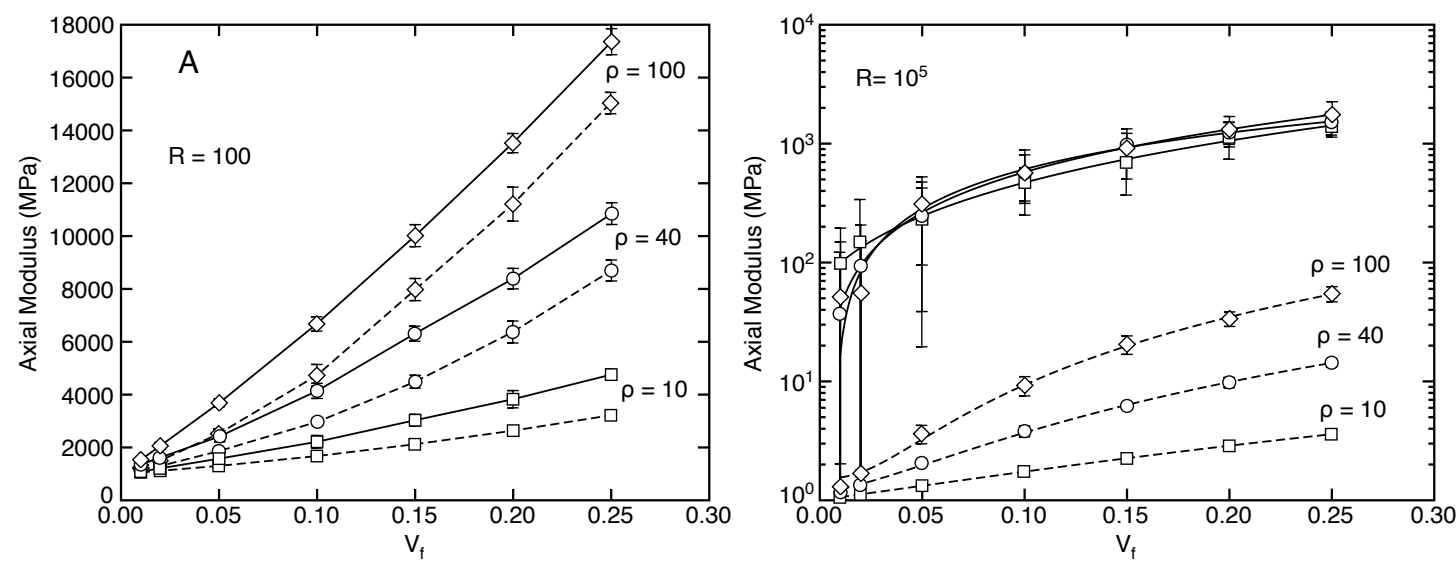

Figure 4: Axial composite modulus for $R=100$ (A) and $R=10^{5}$ (B) for three aspect ratios as a function of fiber volume fraction. The symbols are Monte Carlo BVE results with error bars indicating their standard deviations. The dashed and solid curves connecting the symbols indicate lower and upper bounds, respectively. Note that (A) uses a linear scale while (B) uses a log scale to better visualize all results.

upper bound dropped, while the lower bound was nearly constant. We estimated converged results by extrapolating to zero element size and a discrepancy that was outside errors bars (as calculated by least squares fits with error estimations) remained between upper and lower bound results. In other words, part (probably most) of the difference between stress and strain boundary conditions is that even exact solutions for upper and lower bounds differ. This observation is an effect of the heterogenous BVE. For homogeneous materials, the two boundary conditions used here would give exactly the same results; for heterogeneous materials, however, the effective modulus depends on the boundary conditions. As a consequence, one cannot define the "correct" composite modulus without specifying the boundary conditions as well. The challenge is to determine which results most accurately describe the full-scale composite. This boundary condition effect is a function of $R$. For $R<100$, which comprises the range of most conventional composites, the boundary condition effect is relatively small $(<20 \%)$. But for high $R$, the effect becomes very large (1 to 3 orders of magnitude).

Lastly, we emphasize the BVE bounds are just that — bounds to axial modulus - and should not be construed as a "solution" for modeling composites with high $R$. Indeed, Fig. 5 shows that the upper bound decreases (by a factor of 2) for a refined mesh when $\rho=5$ (we do not know how much it would decrease for large $\rho$ ). Similarly, Fig. 3 shows the upper bound might decrease further if the BVE was made much wider. Nevertheless, the bounds presented here remain as valid bounds to composite properties, even if they are not the best bounds that could ever be obtained. If experimental results 

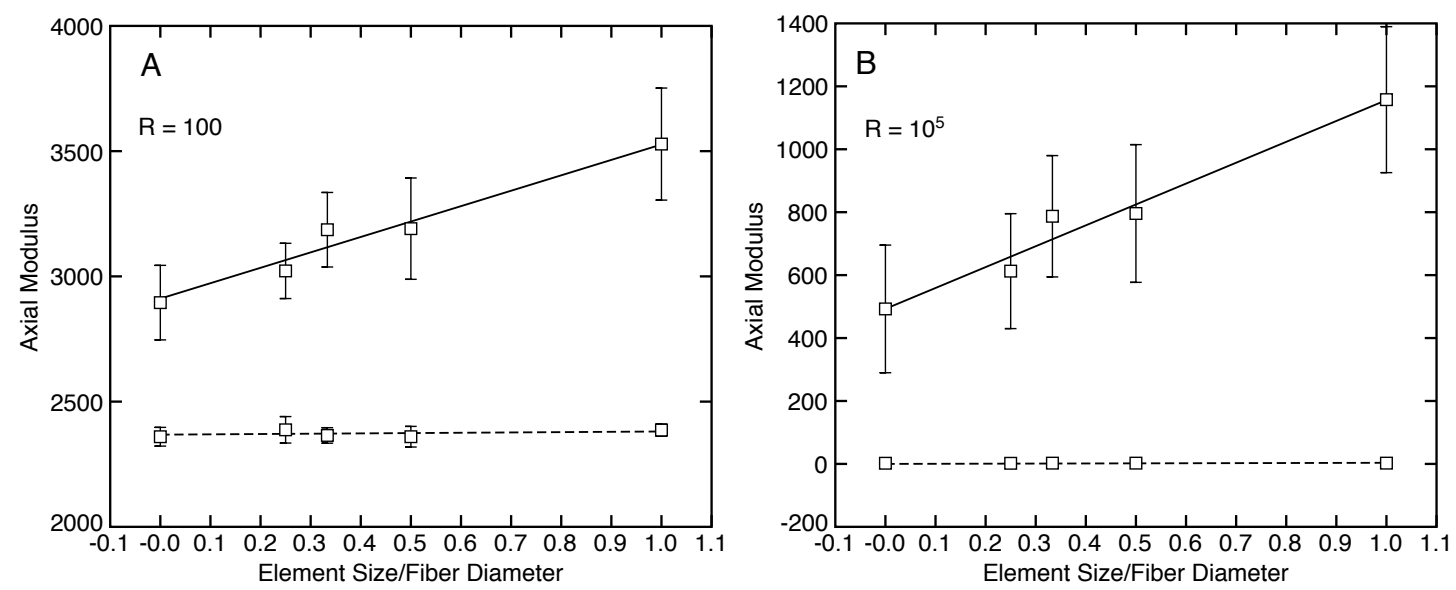

Figure 5: Axial composite modulus for $\rho=5$ and $V_{f}=0.25$ and $R=100$ (A) or $R=10^{5}$ (B) as a function of element size. The lines extrapolate to zero element size or a mesh with an infinite number of elements. The symbols are Monte Carlo BVE results with error bars indicating their standard deviations.

are discovered that exceed these upper bounds, those results potentially indicate a new reinforcement mechanism that needs further study. On the other hand, any experiments bracketed by these bounds may be conventional reinforcement that can be explained by continuum mechanics.

\subsection{How do periodic RVE calculations compare to numerical bounds?}

Most numerical models analyze composite unit cells containing from one or two fibers [9] to many fibers [8]. Figures 6A and B show the two simplest, 2D, periodic structures with parallel or staggered fibers and with dashed lines indicating the smallest repeating unit cells. The parallel structure can be modeled with a single fiber unit cell while the staggered structure requires a unit cell with two complete fibers (each of these can model one quadrant of the unit cell by symmetry). Figure 6C shows a geometrically periodic structure with many fibers subjected to periodic displacement boundary conditions. Models that use any of the unit cells in Figure 6 assume the results reflect the full composite. This section compares results from these periodic structures to the numerical bounds from BVE experiments.

Figure 7 shows the meshes used to model the parallel structure with either rectangular or elliptical fibers (elliptical fiber models were done for comparison to elliptical-fiber-based Eshelby [2] method in the next section). An unknown parameter for an encapsulated fiber is how much matrix is above the fiber and how much is on the sides? We choose this parameter by setting the distance from the fiber end to the top of the mesh, $\Delta$, to equal the distance from the side of the fiber to the edge of the mesh. 


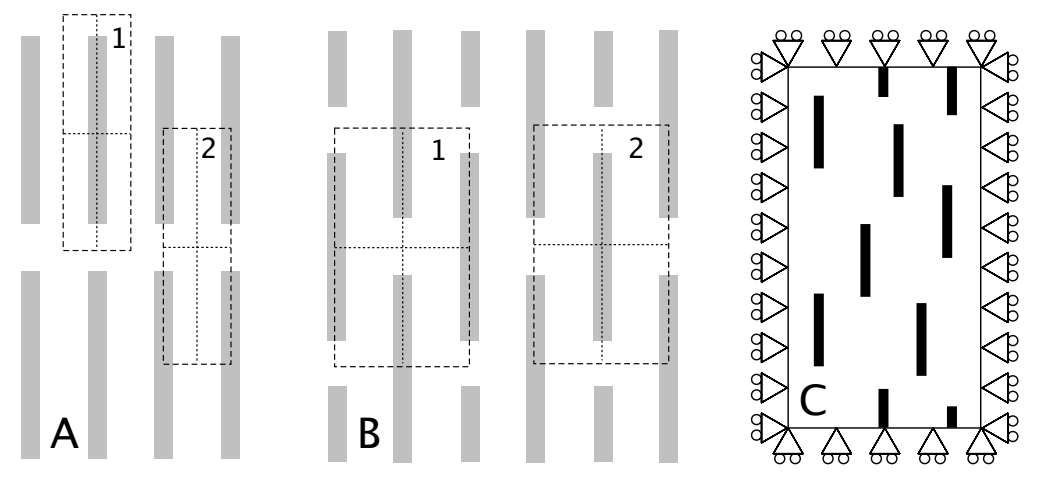

Figure 6: A. A periodic structure with parallel fibers. B. A periodic structure with staggered fibers. C. A geometrically periodic structure with many randomly placed fibers. The dashed lines in A and B outline two possible unit cells for analysis. Each of these has two planes of symmetry allowing the numerical calculations to be done on one quadrant of the cell.

Under this assumption, the rectangular fiber volume fraction is:

$$
V_{f}=\frac{r_{f} L_{f} t}{r_{m} L_{m} t}=\frac{\rho r_{f}^{2}}{\left(r_{f}+\Delta\right)\left(\rho r_{f}+\Delta\right)}
$$

where $t$ is the $2 \mathrm{D}$ thickness. This quadratic equation is easily solved to find $\Delta$ for input modeling parameters $\rho, r_{f}$, and $V_{f}$. For comparing rectangular to elliptical fibers, we kept aspect ratio ( $\rho=$ $\left.L_{f} /\left(2 r_{f}\right)=a / b\right)$ and fiber area (which is also $V_{f}$ in 2D) constant resulting in

$$
2 L_{f} r_{f}=4 \rho r_{f}^{2}=\pi \rho b^{2}=\pi a b \quad \text { or } \quad b=\frac{2 r_{f}}{\sqrt{\pi}} \quad \text { and } \quad a=\frac{2 \rho r_{f}}{\sqrt{\pi}}
$$

where $a$ and $b$ are the major and minor axes of the ellipse. The distance from the top and side of the ellipse to the edge is now found by solving the quadratic equation for $\Delta$ defined from

$$
V_{f}=\frac{\pi a b t}{2 r_{m} L_{m} t}=\frac{\pi \rho b^{2}}{4(b+\Delta)(\rho b+\Delta)}
$$

The meshes for the two fiber unit cells (i.e., staggered structure) were similar, but considered only rectangular fibers. The meshes for the many-fiber periodic structure were regular grids with one element across the fiber width (i.e., same as in BVE calculations) and rectangular fibers only. We did periodic FEA calculations for selected combinations of $R, \rho$, and $V_{f}$ used in the BVE modeling. Unlike the BVE modeling, we were able to refine the one- and two-fiber unit cell meshes and reach convergence by subdividing the elements in Fig. 7 until results became constant (the converged meshes had six elements across the fiber diameter).

First, we analyzed each periodic structure using periodic boundary conditions (e.g., the displacement boundary condition in Fig. 6C) and found modulus from three calculations (analogous to BVE 


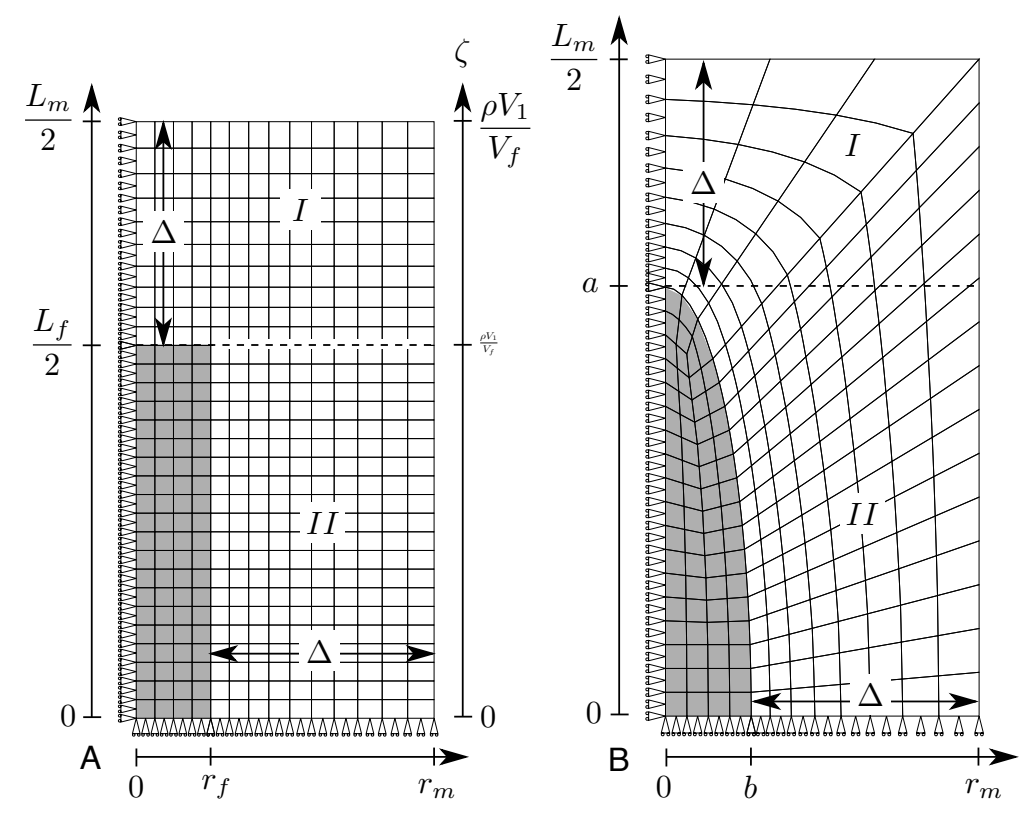

Figure 7: The finite element meshes along with definition of geometric parameters for single-fiber, unit cell models for the parallel structure with either rectangular or elliptical fibers. For clarity, the boundary conditions were omitted and the mesh is shown coarser than the actual mesh used for converged calculations.

method using Eq. (5)). Figure 8A compares these periodic FEA results to BVE bounds. The solid and dashed lines are for parallel structures with rectangular and elliptical fibers, respectively (the dashed elliptical results are mostly obscured by rectangular fiber results). The dash-dot lines are for the staggered structure. The dotted lines with filled triangular symbols are Monte-Carlo FEA of many-fiber unit cells. The open symbols are the BVE bounds. For $R=10$ or 100, the BVE bounds are fairly close and all periodic FEA results were within those bounds. In other words, all methods gave good results. For $R$ greater than 1000, however, the BVE bounds diverge, such as $R=10^{4}$ in Fig. 8A, and all periodic FEA methods track the lower bounds results. The staggered structure (dash-dot lines) was slightly stiffer (as expected) than the parallel structure, but the many-fiber, periodic FEA reverses that trend by reverting to be closer to the lower bound result.

We considered two causes for periodic FEA being lower bound results and far from upper bound results for high $R$. First, the parallel and staggered unit cells were converged results while the BVE results bounds were non-converged results. But, this difference cannot explain the observations, because upper bound BVE results do no converge to lower bound results with a more refined mesh. For example the results in Fig. 5B show that BVE results for $R=10^{5}$ and $\rho=5$ for $V_{f}=0.25$ extrapolated to zero 

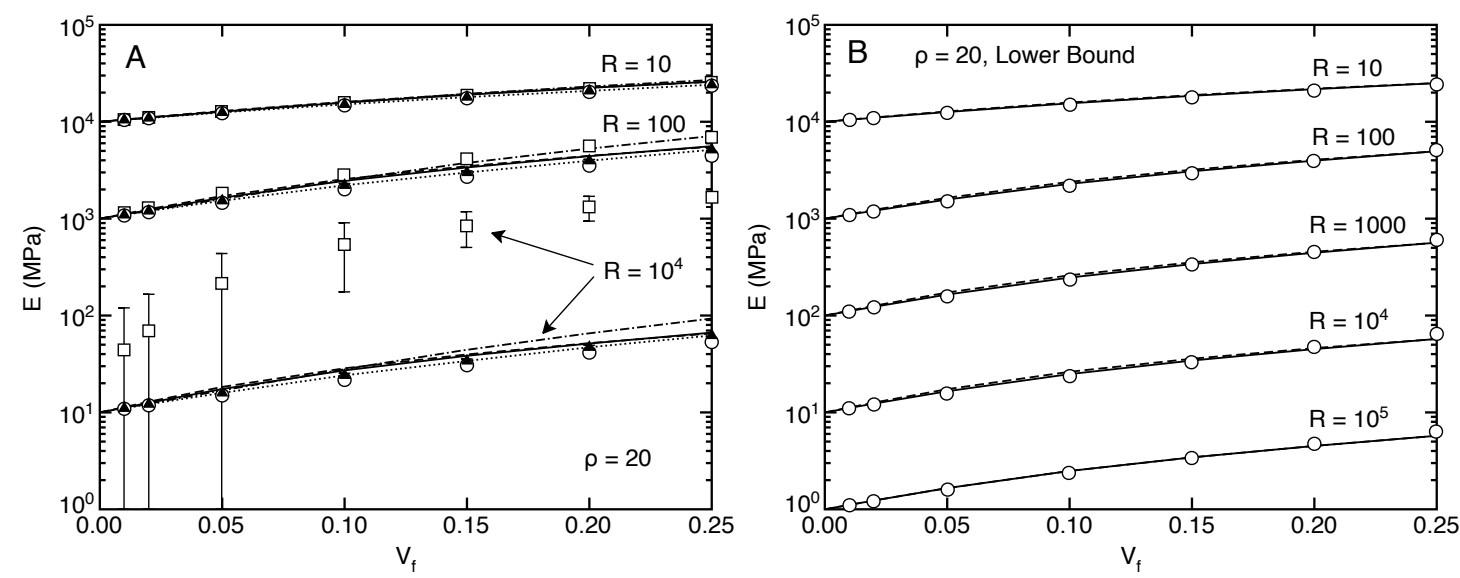

Figure 8: Axial composite modulus for $\rho=20$ as a function of fiber volume fraction for $R$ from 10 to $10^{5}$. A. Compares results of periodic unit cells to BVE bounds. B. Compares fixed traction analysis of the parallel structure to lower bound BVE results. The dashed and solid curves are elliptical and rectangular fibers, respectively. The dashed-dotted lines in A are for numerical analysis of a staggered structure. The filled triangular symbols (with error bars) in A are for Monte Carlo analysis of many-fiber unit cells with periodic boundary conditions. The open symbols (with error bars) are upper bound (squares) and lower bound (circles) BVE results.

element size give upper and lower bound moduli of $492 \pm 202 \mathrm{MPa}$ and $2.15 \pm 0.05 \mathrm{MPa}$, respectively. In contrast, the results for parallel structure with periodic boundary conditions gave $E_{y y}^{(r)}=2.9 \mathrm{MPa}$, which is two orders of magnitude below the upper bound and very close to the lower bound.

Second, we considered the boundary conditions. An alternate approach to periodic boundary conditions is to apply the fixed-strain boundary conditions used for the BVE. This effect clearly can explain the differences. When fixed-strain boundary conditions are applied to the many-fiber unit cell, it is converted essentially into the BVE method and matches the upper bound results. The only difference is the geometrically periodic structure, which as mentioned above has negligible affect on BVE results. The fixed-strain method, however, does not work well for parallel and staggered structures because the final result depends on the choice of unit cell (e.g., quadrants of unit cells 1 or 2 in Fig. 6A and B). This dependence is caused by variable amount of fiber material contacting the fixed-strain boundary conditions. In other words, the BVE method only works for large unit cells (see Fig. 3). A potential concern on all upper bound results is that they are influenced too much by fibers contacting the boundary conditions. It would be a simple matter to artificially force all fibers sufficiently far from the mesh edges. This approach, however, would effectively create a parallel structure that would degenerate to the lower bound results and therefore be far below experimental results. The better solution is to verify the volume element in BVE calculations is sufficiently large and this size requirement may be much 
higher then the size needed when using periodic RVEs with periodic boundary conditions.

Lastly, we comment on rectangular vs. elliptical fibers for the parallel structure. The modulus with a rectangular fiber was always very close to the modulus with an elliptical fiber (as seen by solid and dashed lines in Fig. 8). Looking closer, the elliptical fiber was always slightly stiffer than a rectangular fiber, with the exception of $R \leq 10$ and $\rho<50$. Steif and Hoyson [22] previously looked at cylindrical vs. ellipsoidal fibers and saw larger differences, but they did different calculations. All their results were for the limit of low $V_{f}$ and they used different cylinder-ellipse analogies. Their calculations matched either minor or major axis of the ellipse to the corresponding axis of the cylinder and then either kept $\rho$ or $V_{f}$ constant. We claim our analogy with both $\rho$ and $V_{f}$ constant is more appropriate, although it non-intuitively leads to both the major and minor axes of the ellipse being larger than the fiber length and diameter, respectively. Although rectangular and elliptical fibers gave nearly the same modulus, they gave different fiber stress states. As known from Eshelby, the stress in an elliptical fiber is constant [2]. In contrast, a rectangular fiber shows classic stress transfer with low stress on the ends building to higher stress in the middle. These differences apparently do not affect modulus calculations, but they would be expected to affect models of composite properties that depend on fiber stress, such as modeling of stress transfer or interfacial failure.

\subsection{Can an analytical model sufficiently capture the results of small periodic RVE composites?}

Most analytical models consider a parallel structure with the single fiber geometry in Fig. 7 subjected to constant axial stress in the $y$ direction. Figure 8B shows numerical calculations for the single-fiber unit cell and compares to BVE lower bounds. The numerical calculations used constant traction in the $y$ direction rather then periodic displacement (i.e., the same boundary conditions used in analytical modeling). The numerical results were found to match lower bound results for all values of $V_{f}, \rho$, and $R$ that were studied. Furthermore the modulus for rectangular and elliptical fibers were nearly identical (the solid and dashed lines in Fig. 8B overlap). The next task was to see if analytical modeling reproduces numerical RVE results and thereby inherits agreements with lower bound BVE results, but also disagreements with upper bound BVE results for high $R$.

We first considered an Eshelby analysis [2], but all prior Eshelby models are for 3D composites with ellipsoidal fibers $[1,23]$ where we need a 2D analysis to compare to 2D FEA. Fortunately, the 2D, plane-strain results can be derived as outlined in the Appendix. Although an Eshelby analysis is for low

$V_{f}$, that limit can be removed by a Mori-Tanaka [3] extension. Tucker and Liang [9] point out that a Mori-Tanaka [3] extension can be derived from an Eshelby analysis [2] by replacing the Eshelby tensor, 
$\mathrm{S}$, with an effective tensor, $\mathrm{S}^{*}$, defined by:

$$
\mathrm{S}^{*}=\left(1-V_{f}\right) \mathrm{S}
$$

In addition to an Eshelby/Mori-Tanaka model (E/MT), we also compared to shear-lag methods. The common shear-lag solution in the literature $[4,5]$ and in textbooks $[24,25]$ considers two concentric cylinders (or two parallel layers when in 2D) with zero stress on the fiber ends. We tried this model (using optimal shear-lag parameters [4-7]) and it was inaccurate. For a better analysis, we derived a new shear lag model for a single fiber encapsulated in matrix (i.e., the geometry in Fig. 7A). In addition, the new analysis accounts for stress transfer on the fiber ends and imperfect interfaces between the fiber and the matrix $[7,26]$. This model, denoted as a shear-lag capped model (SLC), is derived next followed by comparison of E/MT and SLC to numerical calculations on the same structure and boundary conditions.

The SLC model is based on recently optimized shear lag methods that alter (and improve) the shear lag parameter [4-6] and explicitly account for imperfect interfaces [7]. The model is split into two regions (see Fig. 7A) and the $y$ axis is converted to a dimensionless coordinate using $\zeta=y / r_{f}$; the fiber top is at $\zeta=\rho$ and the matrix top is at $\zeta=\rho V_{1} / V_{f}$ where $V_{1}$ is fiber volume fraction within region II:

$$
V_{1}=\frac{r_{f} L_{f} t}{r_{m} L_{f} t}=\frac{r_{f}}{r_{f}+\Delta}
$$

where $\Delta$ is found by Eq. (7). Region I is divided into two perfectly-bonded matrix layers aligned with the fiber - a "core" layer from $x=0$ to $r_{f}$ and an "outer" layer from $x=r_{f}$ to $r_{m}$ (note that $V_{1}$ is also core layer volume fraction in region I). The general shear lag solution [5-7] for average axial stress in the core region is:

$$
\left\langle\sigma_{c}(\zeta)\right\rangle=\sigma_{0}+C_{1} e^{\beta_{1} \zeta}+C_{2} e^{-\beta_{1} \zeta}
$$

where $\sigma_{0}$ is the total applied stress and $\beta_{1}$ is the shear lag parameter $[4,5]$ in region I for layers with identical properties and a perfect interface:

$$
\beta_{1}^{2}=\frac{3 G_{m} V_{1}}{E_{m} V_{2}}
$$

Here $V_{2}=1-V_{1}$ is the matrix volume fraction in region II (and outer layer volume fraction in region I) and $E_{m}$ and $G_{m}$ are matrix tensile and shear moduli. Assuming uniform stress $\sigma_{0}$ along the edge at $\zeta=\rho V_{1} / V_{f}$, using force balance, and redefining $C_{1}$, the average axial stresses in the region I layers 
simplify to:

$$
\begin{aligned}
& \left\langle\sigma_{c}(\zeta)\right\rangle=\sigma_{0}+C_{1} \sinh \left(\beta_{1}\left(\zeta-\frac{\rho V_{1}}{V_{f}}\right)\right) \\
& \left\langle\sigma_{o}(\zeta)\right\rangle=\sigma_{0}-\frac{C_{1} V_{1}}{V_{2}} \sinh \left(\beta_{1}\left(\zeta-\frac{\rho V_{1}}{V_{f}}\right)\right)
\end{aligned}
$$

In region II, the average axial stresses in the fiber and matrix are [5-7]:

$$
\left\langle\sigma_{f}(\zeta)\right\rangle=\sigma_{\infty}+C_{3} \cosh \left(\beta_{2} \zeta\right) \text { and }\left\langle\sigma_{m}(\zeta)\right\rangle=\frac{\sigma_{0}-V_{1}\left(\sigma_{\infty}+C_{3} \cosh \left(\beta_{2} \zeta\right)\right)}{V_{2}}
$$

where $\sigma_{\infty}$ is the far-field fiber stress (i.e., stress at the middle of a long fiber) and $\beta_{2}$ is the shear lag parameter in region II [7]:

$$
\beta_{2}^{2}=\frac{\frac{E_{2} V_{1}}{E_{f} E_{m} V_{2}}}{\frac{V_{1}}{3 G_{f}}+\frac{V_{2}}{3 G_{m}}+\frac{V_{1}}{r_{f} D_{t}}}
$$

where $E_{2}=E_{f} V_{1}+E_{m} V_{2}$ is rule of mixtures axial modulus of region II, $E_{f}$ and $G_{f}$ are tensile and shear moduli of the fiber, and $D_{t}$ is an imperfect interface term. The imperfect interface is modeled by allowing interfacial displacement discontinuities that are proportional to the traction in the displacement direction [26]. For region II, the axial displacement jump at $x=r_{f}$ is $[w]=\tau\left(r_{f}\right) / D_{t}$, where $\tau\left(r_{f}\right)$ is the interfacial shear stress. When $D_{t}=\infty$, the displacement jump is zero and the interface is perfect; when $D_{t}=0, \tau\left(r_{f}\right)$ is zero and the interface is debonded; all other $D_{t}$ values model an imperfect interface.

The two unknown constants, $C_{1}$ and $C_{3}$, can be eliminated by continuity conditions between the fiber end and the core layer in region I:

$$
\left\langle\sigma_{f}(\rho)\right\rangle=\left\langle\sigma_{c}(\rho)\right\rangle \quad \text { and } \quad[w(\rho)]=\frac{\left\langle\sigma_{f}(\rho)\right\rangle}{D_{n}}
$$

The first is stress continuity. The second is a new imperfect interface relation on the fiber ends where the jump in axial displacement between the fiber and the core layer is determined by $D_{n}$, which is an imperfect interface parameter analogous to $D_{t}$ but for normal displacements [26]. The displacement jump needed for this condition is calculated from

$$
[w(\rho)]=\Delta\left\langle w_{m}\right\rangle+\Delta\left\langle w_{o}\right\rangle-\Delta\left\langle w_{c}\right\rangle-\Delta\left\langle w_{f}\right\rangle
$$

where $\Delta\left\langle w_{i}\right\rangle$ is the displacement difference between the top and bottom of region i. Using 1D Hooke's 
laws:

$$
\begin{array}{ll}
\Delta\left\langle w_{m}\right\rangle=r_{f} \int_{0}^{\rho}\left(\frac{\left\langle\sigma_{m}(\zeta)\right\rangle}{E_{m}}+\alpha_{m} \Delta T\right) d \zeta & \Delta\left\langle w_{f}\right\rangle=r_{f} \int_{0}^{\rho}\left(\frac{\left\langle\sigma_{f}(\zeta)\right\rangle}{E_{f}}+\alpha_{f} \Delta T\right) d \zeta \\
\Delta\left\langle w_{c}\right\rangle=r_{f} \int_{\rho}^{\frac{\rho V_{1}}{V_{f}}}\left(\frac{\left\langle\sigma_{c}(\zeta)\right\rangle}{E_{m}}+\alpha_{m} \Delta T\right) d \zeta & \Delta\left\langle w_{o}\right\rangle=r_{f} \int_{\rho}^{\frac{\rho V_{1}}{V_{f}}}\left(\frac{\left\langle\sigma_{o}(\zeta)\right\rangle}{E_{m}}+\alpha_{m} \Delta T\right) d \zeta
\end{array}
$$

where $\alpha_{m}$ and $\alpha_{f}$ are the thermal expansion coefficients of the matrix and fiber and $\Delta T$ is the temperature difference. Substituting the stresses, using

$$
\sigma_{\infty}=\frac{E_{f}}{E_{2}} \sigma_{0}+\alpha_{2} \Delta T \quad \text { and } \quad \alpha_{2}=\frac{\alpha_{f} E_{f} V_{1}+\alpha_{m} E_{m} V_{2}}{E_{2}}
$$

where $\alpha_{2}$ is the weighted rule-of-mixtures thermal expansion coefficient of region II, and integrating (in Mathematica, Wolfram Research) gives

where

$$
[w(\rho)]=\frac{r_{f} \rho}{E_{m} V_{2}}\left[2 C_{1} \frac{\sinh ^{2}\left(\beta_{1}^{*} \rho\right)}{\beta_{1} \rho}-C_{3} \frac{E_{2}}{E_{f}} \frac{\sinh \left(\beta_{2} \rho\right)}{\beta_{2} \rho}\right]
$$

$$
\beta_{1}^{*}=\frac{\left(V_{1}-V_{f}\right) \beta_{1}}{2 V_{f}}
$$

Solving Eq. (11) for $C_{1}$ and $C_{3}$ gives:

$$
\begin{aligned}
& C_{1}=\frac{\frac{\sigma_{0}}{2}\left(\frac{E_{m} V_{2} \beta_{1}}{r_{f} D_{n}}+\frac{E_{2} \beta_{1}}{E_{f} \beta_{2}}\left(1-\frac{\sigma_{\infty}}{\sigma_{0}}\right) \tanh \left(\beta_{2} \rho\right)\right) \operatorname{csch}\left(\beta_{1}^{*} \rho\right)}{\sinh \left(\beta_{1}^{*} \rho\right)+\left(\frac{E_{m} V_{2} \beta_{1}}{r_{f} D_{n}}+\frac{E_{2} \beta_{1}}{E_{f} \beta_{2}} \tanh \left(\beta_{2} \rho\right)\right) \cosh \left(\beta_{1}^{*} \rho\right)} \\
& C_{3}=\frac{\sigma_{\infty}\left(\left(\frac{\sigma_{0}}{\sigma_{\infty}}-1\right) \sinh \left(\beta_{1}^{*} \rho\right)-\frac{E_{m} V_{2} \beta_{1}}{r_{f} D_{n}} \cosh \left(\beta_{1}^{*} \rho\right)\right) \operatorname{sech}\left(\beta_{2} \rho\right)}{\sinh \left(\beta_{1}^{*} \rho\right)+\left(\frac{E_{m} V_{2} \beta_{1}}{r_{f} D_{n}}+\frac{E_{2} \beta_{1}}{E_{f} \beta_{2}} \tanh \left(\beta_{2} \rho\right)\right) \cosh \left(\beta_{1}^{*} \rho\right)}
\end{aligned}
$$

This stress state was compared to FEA average stresses and the results were good for a wide range of properties. Although this analysis assumed an isotropic fiber, it works for anisotropic fibers by replacing $E_{f}, G_{f}$, and $\alpha_{f}$ with the corresponding axial properties of an anisotropic fiber.

Finally, the modulus is found by integrating displacements in the outer matrix layers. By this process, the incremental length and effective modulus are:

$$
\Delta L\left(\sigma_{0}, \Delta T\right)=\Delta\left\langle w_{m}\right\rangle+\Delta\left\langle w_{o}\right\rangle \quad \text { and } \quad \frac{1}{E^{*}}=\frac{2 \Delta L\left(\sigma_{0}, 0\right)}{\sigma_{0} L_{m}}
$$

Substituting stresses, $C_{1}$, and $C_{3}$ followed by much simplification (in Mathematica, Wolfram Research), the modulus can be cast as:

$$
\frac{E_{2}}{E^{*}}=1+\left(\frac{E_{f}}{E_{m}}-1\right)\left(V_{1}-V_{f}\right)+\frac{E_{f} V_{f}}{E_{m} V_{m}} \Lambda(\rho)
$$


where

$$
\Lambda(\rho)=\frac{V_{m}}{V_{2}} \frac{\frac{E_{2}}{E_{f}} \frac{\tanh \left(\beta_{1}^{*} \rho\right)}{\beta_{1} \rho}+\left(1+\left(1-\frac{E_{2}}{E_{f}}\right)^{2} \frac{\tanh \left(\beta_{1}^{*} \rho\right)}{\beta_{1} \eta}\right) \frac{\tanh \left(\beta_{2} \rho\right)}{\beta_{2} \rho}}{1+\frac{\tanh \left(\beta_{1}^{*} \rho\right)}{\beta_{1} \eta}+\frac{E_{2}}{\eta E_{f}} \frac{\tanh \left(\beta_{2} \rho\right)}{\beta_{2}}}
$$

and $\eta=E_{m} V_{2} /\left(r_{f} D_{n}\right)$. In the limit of no region I $\left(V_{1} \rightarrow V_{f}\right)$ and debonded fiber end $\left(D_{n} \rightarrow 0\right.$ and $\eta \rightarrow \infty$ to get zero stress on the fiber end), the stresses and modulus reduce to the standard shear lag result for two layers:

$$
C_{3}=-\sigma_{\infty} \operatorname{sech}\left(\beta_{2} \rho\right) \text { and } \frac{E_{2}}{E^{*}}=1+\frac{E_{f} V_{f}}{E_{m} V_{m}} \frac{\tanh \left(\beta_{2} \rho\right)}{\beta_{2} \rho}
$$

The results here extend this old result to an encapsulated fiber including both fiber end stress transfer and an imperfect interface on fiber ends and sides.

Figure 9 compares E/MT and SLC models to numerical single-fiber unit cell (SFUC) models with traction loading and to lower bound BVE results for $R=100$ or $R=10^{4}$ and for $\rho=5,20$, and 70. For both $R=100$ and $R=10^{4}$, both the SLC and E/MT models agreed well with both the SFUC and lower bound BVE. In summary, both the SLC and E/MT models accurately predict lower bound modulus for an aligned, short fiber composite for all tested values of $R$ and $\rho$. They track the lower bound because the models are based on an assumption of uniform far-field stress. Because upper and lower bound results are fairly close for $R \leq 100$, the SLC and E/MT models are also close to upper bound results within this range. But, for $R>100$, these models should be recognized as providing pessimistic, lower bound modulus predictions.

There is a long history of refining analytical models for short fiber composites based on analysis of the single-fiber geometry [9]. Because the SLC and E/MT models agree with refined numerical models for lower bound modulus, this half of the problem is "solved," leaving little room for seeking improved lower bound models. The development of better analytical models for upper bound modulus (e.g., using displacement boundary conditions) is tempting, but we claim is doomed to limited success. The best such analytical modeling could achieve would be to agree with numerical RVE results with periodic boundary conditions. Section 3.2 shows those results also give lower bound results for $R>100$. In summary, future analytical modeling should focus on finding an upper bound modulus using non-unitcell methods.

\subsection{Can an analytical model account for $3 D$ fibers and for imperfect interfaces?}

Because analytical models agree with most lower bound BVE results and with upper bound BVE results for $R \leq 100$, we hypothesize that 3D analytical modeling would agree similarly with 3D BVE 

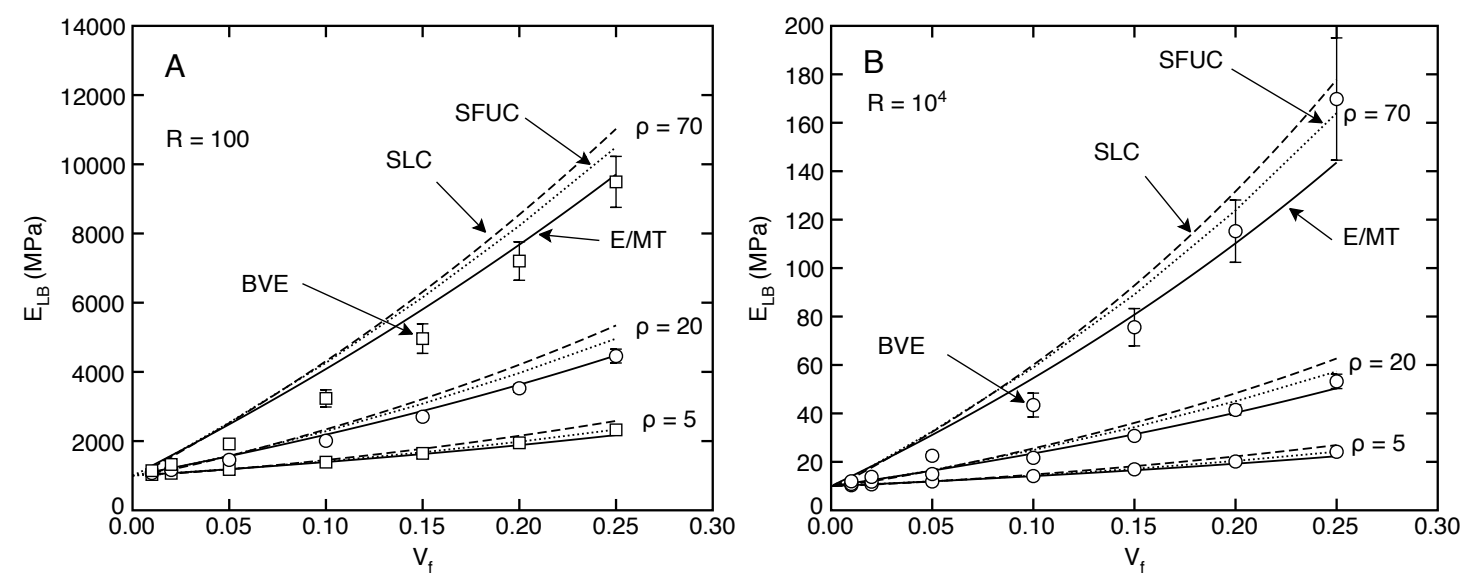

Figure 9: Axial composite modulus for $R=100$ (A) and $R=10^{4}$ (B) as a function of fiber volume fraction for three fiber aspect ratios. The dashed and solid curves are analytical models based on the new shear lag method (SLC) and an Eshelby/Mori-Tanaka approach (E/MT), respectively. The dotted curves are numerical results with traction boundary conditions for a single-fiber unit cell (SFUC). The symbols are lower bound, Monte Carlo BVE results with error bars indicating their standard deviations.

modeling, even though the 3D BVE modeling is not available. This section compares 3D analytical modeling (an axisymmetric analysis), to numerical, axisymmetric, single-fiber unit cell results (which is the only unit cell amenable to axisymmetric analysis). In addition, this section investigates imperfect interface effects $[7,26]$. Unfortunately, an Eshelby analysis [2] cannot be extended to modeling of imperfect interfaces. An Eshelby analysis works by exploiting the observation that elliptical fibers have constant stress; this property allows replacement methods to find modulus $[1,23]$. When the interfaces are imperfect [26], however, the stresses in an elliptical fiber are no longer constant (as confirmed by FEA modeling), which means an Eshelby approach no longer works. In contrast, the SLC model derived above includes imperfect interfaces. This section therefore compares an axisymmetric SLC model to converged, axisymmetric FEA calculations with imperfect interface elements [27] to verify if the SLC model works in 3D and if it correctly models imperfect interfaces.

The SLC analysis in the previous section was for the 2D problem. It can easily be extended to an axisymmetric analysis for an end-capped fiber cylinder simply by redefining volume fractions, $\Delta$, and the shear lag parameters $\beta_{1}$ and $\beta_{2}$. The fiber volume fraction within region II becomes

$$
V_{1}=\frac{\pi r_{f}^{2} L_{f}}{\pi r_{m}^{2} L_{f}}=\frac{\rho r_{f}^{2}}{\left(r_{f}+\Delta\right)^{2}}
$$


where $\Delta$ is found by solving the following cubic equation

$$
V_{f}=\frac{\pi r_{f}^{2} L_{f}}{\pi r_{m}^{2} L_{m}}=\frac{\rho r_{f}^{3}}{\left(r_{f}+\Delta\right)^{2}\left(\rho r_{f}+\Delta\right)}
$$

The shear lag parameters become [7]:

$$
\beta_{1}^{2}=-\frac{4 G_{m} V_{2}}{E_{m}\left(V_{2}+\ln V_{1}\right)} \quad \text { and } \quad \beta_{2}^{2}=\frac{\frac{4 E_{2}}{E_{f} E_{m}}}{\frac{V_{2}}{2 G_{f}}-\frac{1}{G_{m}}\left(\frac{V_{2}}{2}+1+\frac{\ln V_{1}}{V_{2}}\right)+\frac{2 V_{2}}{r_{f} D_{t}}}
$$

After these changes, all other equations in the SLC model are the same.

Figure 10 compares SLC models to FEA analysis of an axisymmetric model using periodic displacement or traction boundary conditions as a function of interface parameter for three different aspect ratios, two $R$ values, and all for $V_{f}=0.15$. In these calculations, the two interface parameters were made equal $D_{n}=D_{t}$ and high values on the right correspond to the perfect interface limit. The SLC model accurately reproduces the numerical results and therefore provides a useful model for studying both 3D (cylindrical fibers) and imperfect interface effects. The SLC model falls between numerical results with displacement and traction boundary conditions and is closer to the traction results. This trend is likely because the SLC model is based on traction boundary conditions. Because an Eshelby analysis [2] cannot account for imperfect interfaces, it is plotted on the right as short, dashed horizontal lines representing the 3D (ellipsoidal fibers), perfect-interface, E/MT result $[1,2,23]$. Contrary to the Russel [23] approach of approximating the Eshelby tensor for high aspect ratio fibers, these calculations used the exact Eshelby tensor for ellipsoidal inclusions given in Ref. [2]. The Eshelby analysis is inconsistent. It is below numerical results for $\rho=5$, but moves above it as $\rho$ increases.

\subsection{Other Properties}

For mean field modeling of random or partially ordered composites, one needs all mechanical properties of the unit cell with random, aligned fibers. Most work focuses on analysis for $E_{y y}^{(r)}$ because it is the property that is most affected by fiber aspect ratio, $\rho$. Christensen [1] shows that for an Eshelby [2] analysis, the shear modulus is independent of $\rho$ and therefore equal to the shear modulus of a continuous fiber composite. Similarly, the transverse modulus, $E_{x x}^{(r)}$, and Poisson's ratio, $v_{y x}$, may only weakly

be affected by $\rho$. In other words, once $E_{y y}^{(r)}$ is found, the remaining properties are assumed to follow by simpler methods. We checked this conventional wisdom with the numerical BVE results. Figure 11 plots $E_{x x}^{(r)}, G_{x y}^{(r)}$, and $v_{y x}^{(r)}$ as a function of $\rho$ for two $R$ values and all for $V_{f}=0.2$. For $R=100$, all these properties are nearly independent of $\rho$ and thus are equal to results for continuous fiber composites. In 

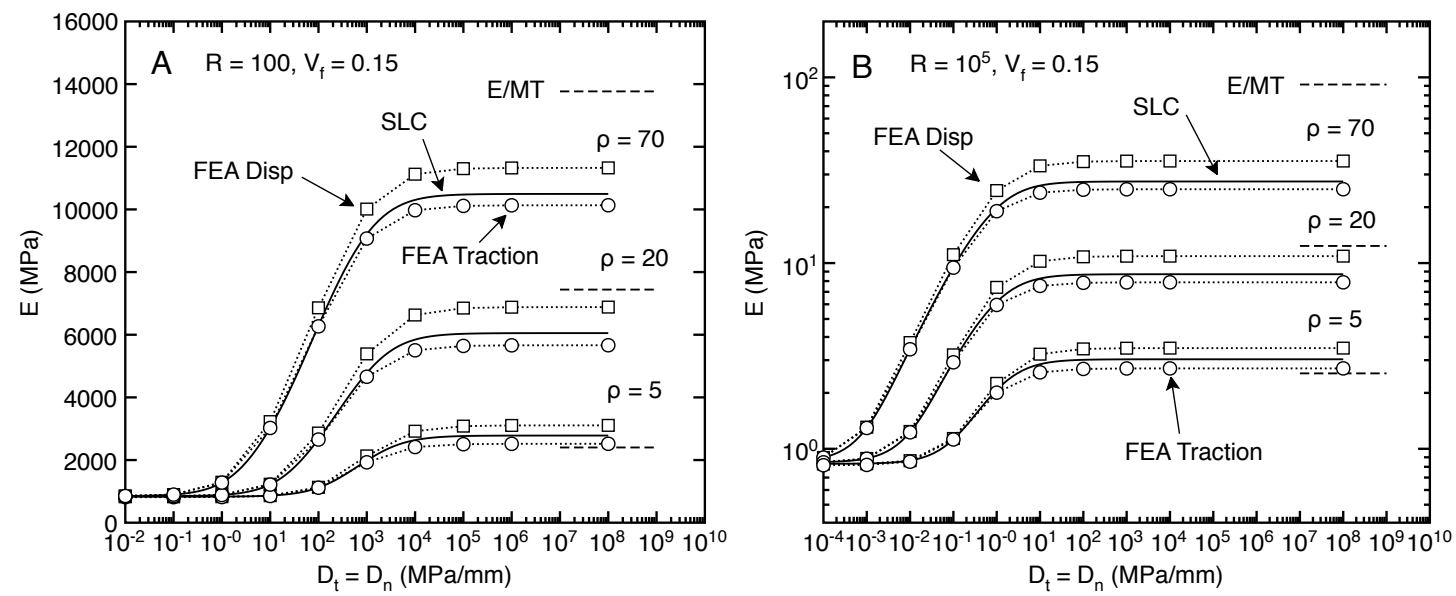

Figure 10: Axial composite modulus for $V_{f}=0.15$ as a function of fiber/matrix imperfect interface parameter and for three fiber aspect ratios. The open and solid symbols are upper and lower bound, axisymmetric, numerical calculations, respectively. The solid curves are axisymmetric version of the new shear lag solution. The dashed lines on the right are 3D Eshelby/Mori-Tanaka approach results for a perfect interface. Note that the $R=10^{5}$ plot uses a log axis.

contrast, for $R=10^{5}, E_{x x}^{(r)}$ and $G_{x y}^{(r)}$ decrease. This behavior will need to be included when doing mean field modeling of such composites.

\section{Conclusions}

This paper tackled seemingly basic questions whose answers, in our opinion, provide insights for evaluating past and future literature on short fiber composites. The novel results were to develop a method for bounding the mechanical properties and use that approach to study a regime that is not commonly examined (namely large $R$ and large $\rho$ ). The numerical experiments showed that for $R<100$, the bounds are rather close and other numerical and analytical models fall within those bounds and therefore must be close to the correct answer. For $R>100$, however, the numerical bounds diverge and both analytical methods and prior numerical methods based on periodic displacement boundary conditions degenerate to lower bound results. We do not claim the numerical upper bounds are predicting the modulus of real materials - they are upper bound results and because of the crude mesh are not the best upper bounds possible. But, all efforts to seek refined upper bounds suggest that at large $R$ even improved upper bounds are two or more orders of magnitude higher than results found by prior methods. Given that some experimental results on reinforcement of elastomers $[14,16]$ exceed predictions by analytical or prior numerical models, but do not exceed upper bound BVE results, the 


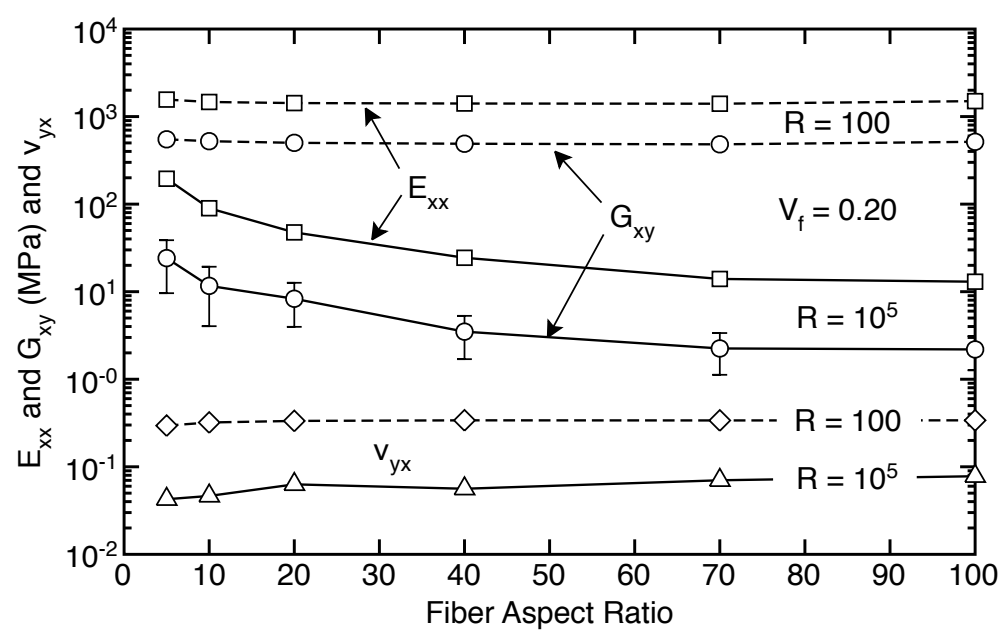

Figure 11: Plots of $E_{x x}^{(r)}, G_{x y}^{(r)}$, and $v_{y x}^{(r)}$ as a function of fiber aspect ratio for two $R$ values and all for $V_{f}=0.2$. The dashed lines are for $R=100$ and the solid lines are for $R=10^{5}$.

two options are that current modeling methods are inadequate or that we must abandon continuum mechanics. The upper bound results here demonstrate the answer might be the former.

Analytical models typically treat a single fiber. The Eshelby/ Mori-Tanaka approach [2, 3] and the new shear lag method presented here, agreed well with lower bound, numerical, single-fiber models, but gave no information about upper bound modulus for high $R$. The new shear lag method adds modeling for imperfect interfaces. It is not worth the effort to seek "improved" analytical models because there is little room to improve agreement with numerical modeling on the same structure. The more interesting problem is how to avoid degenerating to a lower bound results. The next tasks for short fiber composite modeling should be to develop a new approach to analytical modeling that can predict upper bound moduli for all values of $R$ and to extend that modeling to composites with non-aligned fibers by averaging over a fiber distribution function [1].

\section{Acknowledgements}

This work was support, in part, by the National Science Foundation grant CMMI 1161305 and by the USDA Forest Products Lab under 11-JV-11111129-137. We thank an anonymous reviewer for many insights that lead to significant revisions and hopefully an improved presentation. 


\section{Appendix}

A 2D, plane-strain Eshelby analysis [2] can be derived by following Christensen [1], which is based on Russel [23]. We begin by specifying $\varepsilon_{11}=\varepsilon_{0}, \varepsilon_{22}=\varepsilon_{r}$, and $\varepsilon_{33}=0$, where the fiber is the 1 direction and the 3 direction is the thickness direction with zero strain for a plane-strain analysis. Modifying Russel [23] with a plane-strain constitutive law and requiring only the stress in the 2 direction to be zero, the effective fiber direction modulus and Poisson's ratio become:

$$
E_{11}^{*}=E_{m}^{(r)}\left(1-V_{f}\left(A_{1111}-A_{1122} v_{12}^{*}\right)\right) \quad \text { and } \quad v_{12}^{*}=\frac{v_{m}^{(r)}-V_{f}\left(A_{2211}+v_{m}^{(r)} A_{1111}\right)}{1-V_{f}\left(A_{2222}+v_{m}^{(r)} A_{1122}\right)}
$$

where $A_{i j k l}$ are elements of the fourth-rank tensor that relates Eshelby transformation strain to applied strain:

$$
\varepsilon_{i j}^{T}=A_{i j k l} \varepsilon_{k l}^{0}
$$

Continuing along a 2D plane-strain analog of the Russel analysis [23], the $A_{i j k l}$ terms can be found from

$$
\begin{aligned}
A_{1111}=\frac{a_{22} b_{11}-a_{12} b_{12}}{a_{11} a_{22}-a_{12} a_{21}} & A_{1122}=\frac{a_{22} b_{12}-a_{12} b_{22}}{a_{11} a_{22}-a_{12} a_{21}} \\
A_{2211}=\frac{a_{11} b_{12}-a_{21} b_{11}}{a_{11} a_{22}-a_{12} a_{21}} & A_{2222}=\frac{a_{11} b_{22}-a_{21} b_{12}}{a_{11} a_{22}-a_{12} a_{21}}
\end{aligned}
$$

where

$$
\begin{aligned}
& a_{11}=\Delta \lambda\left(S_{1111}+S_{2211}\right)+2 \Delta G S_{1111}+\lambda_{m}+2 G_{m} \\
& a_{12}=\Delta \lambda\left(S_{1122}+S_{2222}\right)+2 \Delta G S_{1122}+\lambda_{m} \\
& a_{21}=\Delta \lambda\left(S_{1111}+S_{2211}\right)+2 \Delta G S_{2211}+\lambda_{m} \\
& a_{22}=\Delta \lambda\left(S_{1122}+S_{2222}\right)+2 \Delta G S_{2222}+\lambda_{m}+2 G_{m} \\
& b_{11}=b_{22}=-\Delta \lambda-2 \Delta G=-\left(\lambda_{f}-\lambda_{m}\right)-2\left(G_{f}-G_{m}\right) \\
& b_{12}=b_{21}=-\Delta \lambda=-\left(\lambda_{f}-\lambda_{m}\right)
\end{aligned}
$$

and $S_{i j k l}$ are elements of the 2D, plain-strain Eshelby tensor. The terms $\lambda_{f}$ and $\lambda_{m}$ are the Lamé parameters for the fiber and matrix. For results, Russel [23] substituted the 3D Eshelby tensor [2] (as approximated for large aspect ratio $[1,23]$ ) and took limiting results for small $V_{f}$. Here we need the 2D plane-strain Eshelby tensor [2] evaluated exactly to handle small aspect ratios and we used the full 
modulus and Poisson's ratio expressions instead of their low $V_{f}$ limits. Fortunately, Eshelby [2] provides explicit 2D plane-strain results for the case with elliptical axes $a>b(\rho=a / b)$ and $c=\infty$ :

$$
\begin{aligned}
& S_{1111}=\frac{1}{2\left(1-v_{m}\right)}\left(\frac{1+2 \rho}{(\rho+1)^{2}}+\frac{1-2 v_{m}}{\rho+1}\right) \\
& S_{1122}=\frac{1}{2\left(1-v_{m}\right)}\left(\frac{1}{(\rho+1)^{2}}-\frac{1-2 v_{m}}{\rho+1}\right) \\
& S_{2211}=\frac{1}{2\left(1-v_{m}\right)}\left(\frac{\rho^{2}}{(\rho+1)^{2}}-\frac{\left(1-2 v_{m}\right) \rho}{\rho+1}\right) \\
& S_{2222}=\frac{1}{2\left(1-v_{m}\right)}\left(\frac{\rho(\rho+2)}{(\rho+1)^{2}}+\frac{\left(1-2 v_{m}\right) \rho}{\rho+1}\right)
\end{aligned}
$$

Here $v_{m}$ is the unreduced matrix Poisson's ratio and it is related to the plane-strain Poisson's ratio by $v_{m}=v_{m}^{(r)} /\left(1+v_{m}^{(r)}\right)$.

\section{References}

[1] R. M. Christenson, Mechanics of Composite Materials, John Wiley \& Sons, New York, 1979.

[2] J. D. Eshelby, The determination of the elastic field of an ellipsoidal inclusion and related problems, Proc. R. Soc. Lond. A 241 (1957) 376-396.

[3] T. Mori, K. Tanaka, Average stress in matrix and average elastic energy of materials with misfitting inclusions, Acta Metallurgica 21 (1973) 571-574.

[4] L. N. McCartney, Analytical models of stress transfer in unidirectional composites and cross-ply laminates, and their application to the prediction of matrix/transverse cracking, in: J. N. Reddy, K. L. Reifsnider (Eds.), Proc. IUTAM Symposium, Blacksburg, VA, 1991, 1992, pp. 251-282.

[5] J. A. Nairn, On the use of shear-lag methods for analysis of stress transfer in unidirectional composites, Mech. of Materials 26 (1997) 63-80.

[6] J. A. Nairn, D.-A. Mendels, On the use of planar shear-lag methods for stress-transfer analysis of multilayered composites, Mechanics of Materials 33 (2001) 335-362.

[7] J. A. Nairn, Generalized shear-lag analysis including imperfect interfaces, Advanced Composite Letters 13 (2004) 263-274.

[8] A. A. Gusev, Representative volume element size for elastic composites: A numerical study, Journal of the Mechanics and Physics of Solids 45 (9) (1997) 1449 - 1459. 
[9] C. L. Tucker, E. Liang, Stiffness predictions for unidirectional short-fiber composites: Review and evaluation, Composite Science and Technology 59 (1999) 655-671.

[10] A. A. Gusev, Numerical identification of the potential of whisker- and platelet-filled polymers, Macromolecules 34 (9) (2001) 3081-3093.

[11] H. Bohm, A. Eckschlager, W. Han, Multi-inclusion unit cell models for metal matrix composites with randomly oriented discontinuous reinforcements, Computational Materials Science 25 (1-2) (2002) $42-53$.

[12] H. R. Lusti, A. A. Gusev, Finite element predictions for the thermoelastic properties of nanotube reinforced polymers, Modelling and Simulation in Materials Science and Engineering 12 (3) (2004) S107.

[13] E. Ghossein, M. Lévesque, A comprehensive validation of analytical homogenization models: The case of ellipsoidal particles reinforced composites, Mechanics of Materials 75 (2014) 135-150.

[14] V. Favier, S. C. S. G. R. Canova, J.-Y. Cavaille, Mechanical percolation in cellulose whisker nanocomposites, Polymer Engineering and Science 37 (1997) 1732-1739.

[15] M. D. Frogley, D. Ravich, H. Wagner, Mechanical properties of carbon nanoparticle-reinforced elastomers, Composites Science and Technology 63 (11) (2003) 1647 - 1654, modeling and Characterization of Nanostructured Materials.

[16] J. R. Capadona, K. Shanmuganathan, D. J. Tyler, S. J. Rowan, C. Weder, Stimuli-Responsive Polymer Nanocomposites Inspired by the Sea Cucumber Dermis, Science 319 (5868) (2008) 13701374.

[17] F. Deng, M. Ito, T. Noguchi, L. Wang, H. Ueki, K.-i. Niihara, Y. A. Kim, M. Endo, Q.-S. Zheng, Elucidation of the reinforcing mechanism in carbon nanotube/rubber nanocomposites, ACS Nano 5 (5) (2011) 3858-3866.

[18] J. A. Nairn, Material point method (NairnMPM) and finite element analysis (NairnFEA) opensource software, http://code.google.com/p/nairn-mpm-fea/ (2014).

[19] Z. Hashin, S. Shtrikman, On some variational principles in anisotropic and nonhomogeneous elasticity, J. Mech. Phys. Solids 10 (1962) 335-342. 
[20] Z. Hashin, Elasticity of random media, Transactions of the Society of Rheology 9 (1) (1965) 381-406.

[21] Z. Hashin, Mechanics of Composite Materials, Pergamon Press, 1969, Ch. Theory of Composite Materials, pp. 201-242.

[22] P. S. Steif, S. F. Hoyson, An energy method for calculating the stiffness of aligned short-fiber composites, Mechanics of Materials 6 (1987) 197-210.

[23] W. B. Russel, On the effective moduli of composite materials: Effect of fiber length and geometry at dilute concentrations, Z. Angew. Math. Phys. 24 (1973) 581-600.

[24] M. R. Piggott, Load Bearing Fiber Composites, Pergamon Press, Oxford, UK, 1980.

[25] D. Hull, T. W. Clyne, An Introduction to Composite Materials, 2nd Edition, Cambridge University Press, 1996.

[26] Z. Hashin, Thermoelastic properties of particulate composites with imperfect interface, Journal of the Mechanics and Physics of Solids 39 (6) (1991) 745-762.

[27] J. A. Nairn, Numerical implementation of imperfect interfaces, Computational Materials Science 40 (2007) 525-536. 